\title{
Role of Tc-99m MIBI in the evaluation of single pulmonary nodules: a preliminary report
}

\author{
Omar A Minai, Shankar Raja, Atul C Mehta, Eugene J Sullivan, Saeed U Khan, \\ Asok Dasgupta, Alejandro C Arroliga
}

\begin{abstract}
Background-Survival in bronchial carcinoma is closely related to the stage of the disease at the time of diagnosis and a single pulmonary nodule represents a potentially curable stage. This study was conducted to assess the feasibility of using Tc-99m labelled 2-methoxy isobutyl isonitrile (MIBI) to differentiate benign from malignant single pulmonary nodules.

Methods-A prospective study was conducted in the outpatient pulmonary clinic at the Cleveland Clinic Foundation. Twenty five patients with single pulmonary nodules considered indeterminate by their physicians and undergoing a procedure for tissue diagnosis were evaluated by Tc-99m MIBI SPECT scanning prior to definitive testing. Assessment of MIBI uptake was done qualitatively (subjectively) and quantitatively and correlated with the histopathology and nodule size.

Results-Of the 21 patients with malignant lesions, 18 had increased uptake of MIBI corresponding to the location of the nodule and were considered positive. The predominant tumour types were large cell $(n=5)$ and adenocarcinoma $(n=10)$. All four patients with benign lesions had negative MIBI scans. For malignancy the overall specificity was $100 \%$, sensitivity was $85.7 \%$, positive predictive value was $100 \%$, and negative predictive value was $\mathbf{5 7 \%}$. Quantitative uptake of MIBI correlated with the diameter of the nodule with a correlation coefficient of 0.61 by Spearman's rank sum test. This relationship was statistically significant $(p=0.02)$. Conclusion-This preliminary study suggests that Tc-99m MIBI has a very high specificity and positive predictive value for malignant single pulmonary nodules and might be a useful non-invasive diagnostic modality in their management. (Thorax 2000;55:60-62)
\end{abstract}

Keywords: single pulmonary nodules; Tc-99m MIBI SPECT scanning; lung cancer

Bronchial carcinoma is one of the most prevalent and aggressive malignancies. The American Cancer Society estimates that 172000 cases of bronchial carcinoma will be reported in 1998 in the USA, ${ }^{1} 30 \%$ of which will present as solitary lesions in the lung. Survival in bronchial carcinoma is closely related to the stage of the disease at the time of diagnosis and a single pulmonary nodule represents a potentially curable stage.

Evaluation of such lesions represents a frequent vexing dilemma for clinicians. Invasive procedures are often undertaken to obtain a specific tissue diagnosis because none of the currently available non-invasive diagnostic modalities affords accurate differentiation between benign and malignant nodules. ${ }^{2}$ Stable nodule size for more than two years, the presence of fat within the nodule, and characteristic patterns of calcification (concentric, central or stippled) are the only radiographic criteria currently accepted as suggestive of a benign lesion. ${ }^{2}$

Various radionuclides have been tried to differentiate benign from malignant pulmonary nodules. Positron emission tomography (PET) using 18-fluorodeoxyglucose (FDG) is helpful but the expense and limited availability have restricted its use. Increased uptake of technetium-99m hexakis 2-methoxy isobutyl isonitrile (Tc-99m MIBI) in bronchogenic carcinoma on single photon emission tomographic scanning (SPECT) has been reported. ${ }^{3}$ This study was conducted to evaluate the feasibility of employing this technique in differentiating benign from malignant pulmonary nodules.

\section{Methods}

PATIENTS

Patients were included if the chest radiograph showed a single nodule less than $6 \mathrm{~cm}$ in diameter completely surrounded by lung tissue, with or without hilar and/or mediastinal lymphadenopathy, which was considered indeterminate by clinical and radiographic criteria. Only patients in whom a procedure to obtain definitive tissue diagnosis was planned were included in the study. A thoracic computed tomographic (CT) scan was performed if deemed appropriate by the primary physician and was interpreted independently. SPECT scanning was performed before any definitive diagnostic procedure.

SPECT SCAN

Tc-99m MIBI, 20-25 mCi, was injected intravenously and SPECT images were acquired in 
Table 1 Radiographic and histopathological features of the pulmonary nodules

\begin{tabular}{llllll}
\hline $\begin{array}{l}\text { Case } \\
\text { no. }\end{array}$ & Location & $\begin{array}{l}\text { Nodule size } \\
\text { (cm) }\end{array}$ & $\begin{array}{l}\text { Diagnostic } \\
\text { procedure }\end{array}$ & Pathology & $\begin{array}{c}\text { MIBI SPECT } \\
+/-\end{array}$ \\
\hline 1 & LUL & 1.4 & OLBx & Adenocarcinoma & + \\
2 & LLL & 2.5 & TTNA & Adenocarcinoma & + \\
3 & RML & 2.0 & TTNA & Large cell carcinoma & + \\
4 & RUL & 1.9 & OLBx & Small cell carcinoma & + \\
5 & LUL & 2.0 & OLBx & Adenocarcinoma & + \\
6 & RUL & 2.3 & OLBx & Adenocarcinoma & + \\
7 & RML & 1.6 & OLBx & Non-small cell carcinoma & + \\
8 & RUL & 1.6 & TTNA & Adenocarcinoma & + \\
9 & LUL & 2.5 & OLBx & Non-small cell carcinoma & + \\
10 & RLL & 5.0 & OLBx & Large cell carcinoma & + \\
11 & LUL & 4.0 & OLBx & Large cell carcinoma & + \\
12 & RUL & 3.0 & TTNA & Adenocarcinoma & + \\
13 & RLL & 1.1 & OLBx & Large cell carcinoma & + \\
14 & LLL & 3.0 & OLBx & Large cell carcinoma & + \\
15 & RUL & 4.5 & OLBx & Bronchoalveolar cell & + \\
& & & & carcinoma & + \\
16 & RUL & 5.0 & TTNA & Adenocarcinoma & + \\
17 & RUL & 1.5 & OLBx & Bronchoalveolar cell & + \\
& & & & carcinoma & \\
18 & RUL & 2.5 & FB & Sarcoma & + \\
19 & RUL & 1.3 & FB & Adenocarcinoma & - \\
20 & LLL & 3.0 & FB & Squamous cell carcinoma & - \\
21 & RLL & 1.2 & OLBx & Squamous cell carcinoma & - \\
22 & RML & 1.5 & OLBx & Histoplasamosis & - \\
23 & RUL & 2.5 & FB & Tuberculosis & - \\
24 & LUL & 2.3 & OLBx & Granuloma & - \\
25 & LUL & 1.5 & OLBx & Granuloma & - \\
\hline
\end{tabular}

$\mathrm{FB}=$ flexible bronchoscopy; $\mathrm{LUL}=$ left upper lobe $\mathrm{LLL}=$ left lower lobe OLBx $=$ open lung biopsy; RUL = right upper lobe; RML = right middle lobe; RLL = right lower lobe; TTNA = transthoracic needle aspiration.

all patients within 5-10 minutes of injection on a dual headed gamma camera (BIAD, Trionix; Twinsburg, Ohio, USA). Projection data were acquired for 25 minutes and the raw data were processed using routine back projection algorithms. Reconstructed images were evaluated in two ways: (1) qualitatively (subjective visual evaluation) for abnormal accumulation of Tc-99m MIBI corresponding to the location of the nodule on the chest radiograph or CT scan and (2) quantitatively by comparing uptake of MIBI in the nodule (T) with (a) uptake at a corresponding point in the opposite lung (OL) and (b) uptake in "normal" lung (NL), expressed as ratios (T/OL and T/NL). "Normal" lung was taken as the area of lung felt to be normal by a radiologist on a chest radiograph. The SPECT reader (SR) was blinded to the final diagnosis.
Table 2 Qualitative assessment of MIBI SPECT scan

\begin{tabular}{lll}
\hline & $\begin{array}{l}\text { Malignancy } \\
\text { positive }(n=21)\end{array}$ & $\begin{array}{l}\text { Malignancy } \\
\text { negative }(n=4)\end{array}$ \\
\hline MIBI SPECT positive & 18 & 0 \\
MIBI SPECT negative & 3 & 4 \\
\hline
\end{tabular}

Table 3 Quantitative assessment of MIBI uptake in malignant nodules $(n=14)$

\begin{tabular}{lll}
\hline Parameter & $T / O L^{\star}$ & $T / N L^{\star *}$ \\
\hline Mean (SD) & $1.86(0.67)$ & $2.15(0.80)$ \\
Range & $1.21-3.66$ & $1.37-4.13$ \\
Correlation coefficient $\dagger$ & 0.61 & 0.64 \\
p value & $0.02 \ddagger$ & $0.01 \ddagger$ \\
\hline
\end{tabular}

${ }^{\star} \mathrm{T} / \mathrm{OL}=$ ratio of nodule uptake to uptake of MIBI by opposite lung.

$\star \star \mathrm{T} / \mathrm{NL}=$ ratio of nodule uptake to uptake of MIBI by "normal" lung.

†Correlation of nodule size and MIBI uptake ratios by Spearman's rank sum test.

$\ddagger$ Statistically significant.

The study was approved by the institutional review board and all patients gave informed consent.

\section{Results}

Twenty five patients (11 men) of mean (SD) age $66.8(10.9)$ years with a pulmonary nodule over a one year period were included in the study. Table 1 presents the radiographic and histopathological features of each case. The median nodule size was $2.15 \mathrm{~cm}$, range 1.1$5.0 \mathrm{~cm}$, mean (SD) $2.38(1.14) \mathrm{cm}$. Four nodules were larger than $3.0 \mathrm{~cm}$ in diameter. Most of the tumours were adenocarcinomas $(n=10$, including two bronchoalveolar cell carcinomas) or large cell carcinomas $(n=5)$. Of the 21 patients with malignant lesions, 18 had positive scans by qualitative assessment (fig $1 \mathrm{~A}$ and $\mathrm{B}$ ). There were four patients with benign granulomatous lesions, all of whom had negative MIBI scans. Qualitative assessment of MIBI SPECT scans (table 2 ) had a $100 \%$ specificity and positive predictive value for malignancy with a sensitivity of $85.7 \%$ and a negative predictive value of $57 \%$. Although not specifically looked at-that is, in comparison with CT scans and pathology-increased MIBI uptake was noted
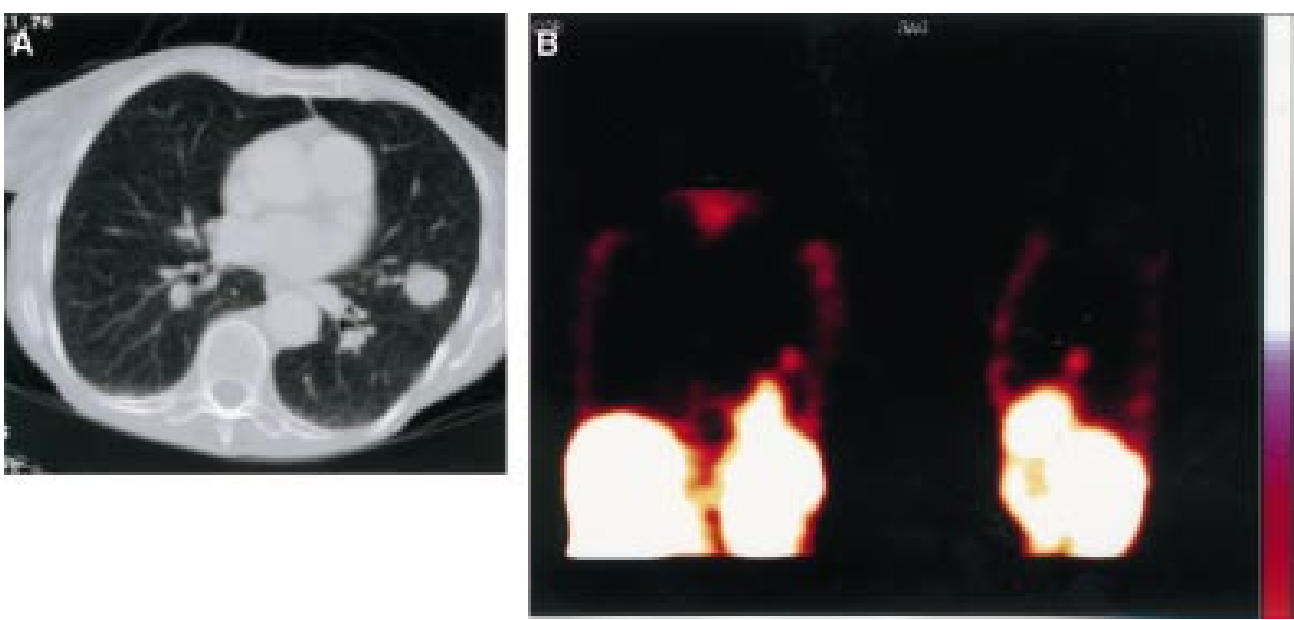

Figure 1 (A) CT scan of the chest revealing a $3 \mathrm{~cm}$ nodule in the left lower lobe of the lung. (B) Tc-99m MIBI SPECT scan in the same patient showing increased uptake of MIBI in the region of the nodule. At surgery the nodule was found to be a large cell carcinoma. 
in mediastinal lymph nodes in four of 21 patients with malignant lesions; only one patient had increased uptake in the hilar lymph nodes. None of the patients with benign lesions had increased MIBI uptake in the lymph nodes.

Quantitative assessment of the four benign nodules was not performed as the lesions could not be identified on MIBI scans and were thus taken to have $\mathrm{T} / \mathrm{OL}$ and $\mathrm{T} / \mathrm{NL}$ ratios of 1.0 . Of the 18 positive scans in patients with malignant lesions, archived data to perform quantitative assessment were available in 14. The mean (SD) ratios for $\mathrm{T} / \mathrm{OL}$ and $\mathrm{T} / \mathrm{NL}$ were 1.86 (0.67) and 2.15 (0.80), respectively (table 3 ). Quantitative uptake of MIBI (T/OL) correlated with the diameter of the nodules with a correlation coefficient of $0.61(p=0.02)$ by Spearman's rank sum test (table 3).

\section{Discussion}

The ideal imaging modality in single pulmonary nodules should differentiate between benign and malignant aetiologies with an acceptable sensitivity and specificity. Despite the use of advanced CT techniques such as contrast enhancement, high resolution CT scanning, densitometry using reference phantoms and spiral CT scans, a significant number of single pulmonary nodules remain indeterminate. ${ }^{5}$ Of the available radionuclides only thallium-201 and gallium-67 are used for this purpose. ${ }^{4}$ Thallium201 suffers from a lack of specificity due to uptake in granulomatous inflammation such as tuberculosis and sarcoidosis. ${ }^{4}$

Technetium-99m MIBI, a lipophilic cation primarily used for myocardial perfusion imaging, has also been used for visualisation of tumours in the lungs, ${ }^{3}$ central nervous system, bones, and thyroid. Although the exact mechanism of increased Tc-99m MIBI uptake in tumour cells is not understood, it has been suggested that it may be related to factors such as passive diffusion, plasma membrane electrical potentials, mitochondrial index, and the lipophilic nature of MIBI. ${ }^{6}{ }^{7}$ Muller et al $l^{8}$ were the first to describe increased uptake of MIBI in bronchogenic carcinoma. Studies to differentiate between benign and malignant lung lesions based on intensity of uptake have shown mixed results. ${ }^{39-12}$

This study suggests that Tc-99m MIBI SPECT scanning has a high specificity for malignancy. The clinical implication of the finding of a very high specificity and positive predictive value is that, in the case of a single pulmonary nodule whose nature is indeterminate after non-invasive evaluation, a positive Tc-99m MIBI scan may help to avoid unnecessary invasive diagnostic testing and allow the physician to proceed directly to thoracotomy. In addition, it may be helpful in cases where non-invasive testing is suspicious for malignancy but the patient has impaired cardiopulmonary function such that the risks of thoracotomy are judged to be high. A positive MIBI SPECT scan would increase the patient's and physician's "level of comfort" that surgical intervention is necessary and worth the risk.

This study shows a low negative predictive value which may be due in part to a low preva- lence of benign lesions in our patient population. This, however, will be true of any patient population that is selected for patients with nodules that are considered indeterminate or possibly malignant by non-invasive criteria. In this study three of 21 malignant nodules were negative by MIBI SPECT scanning. Two of these were among the smallest in the study, measuring 1.2 and $1.3 \mathrm{~cm}$ in diameter which is below the resolution of our scanner (optimal resolution 1.5$2.0 \mathrm{~cm}$ ). The larger nodule $(3.0 \mathrm{~cm}$ ) was an undifferentiated squamous cell carcinoma. It has previously been reported that undifferentiated squamous cell carcinomas can be negative by MIBI SPECT scanning. ${ }^{3}$ Nishiyama et $a l^{11}$ found that squamous cell carcinomas had lower $\mathrm{T} / \mathrm{NL}$ ratios $(2.5(0.9))$ than adenocarcinomas (2.7 (1.4)) and small cell carcinomas (3.0 (1.1)). The low negative predictive value in this study suggests that, if the scan is negative, further work up is needed to establish the final diagnosis.

MIBI uptake ratios (T/OL and T/NL) showed a statistically significant positive correlation with nodule size which means that larger nodules will be easier to identify on MIBI scans. Although no cut off size was identified in our study, two of the three false negative nodules were among the smallest in the study.

More data are needed from larger series to develop decision making protocols based upon MIBI results. Easy availability and lower cost (compared with positron emission tomography), coupled with its high specificity and positive predictive value, make it attractive as a diagnostic modality.

We conclude that, due to its high specificity and positive predictive value, Tc-99m MIBI SPECT imaging can be helpful in the evaluation of single pulmonary nodules. Further studies with larger numbers of patients are required to better delineate its impact on clinical decision making.

1 Landis SH, Murray T, Bolden S, et al. Cancer statistics, 1998. Cancer f Clin 1998;48:6-29.

2 Cummings SR, Lillington GA, Richard RJ. Estimating the probability of malignancy in solitary pulmonary nodules: a Bayesian approach. Am Rev Respir Dis 1986;134:449-52.

Bayesian approach. Am Rev Respir Dis 1986; 134:449-52.
3 Hassan IM, Sahweil A, Constantinides C, et al. Uptake and kinetics of Tc- $99 \mathrm{~m}$ hexakis 2-methoxyisobutylisonitrile in benign and malignant lesions in the lungs. Clin Nucl Med 1989;14:333-40.

4 Abdel-Dayem HM, Scott A, Macapinlac H, et al. Tracer imaging in lung cancer. Eur f Nucl Med 1994;21:57-81.

5 Cortese DA. Solitary pulmonary nodule: observe, operate or what? Chest 1982;81:662-3.

6 Chiu ML, Kronauge JF, Piwnica-Worms D. Effect of mitochondrial and plasma membrane potentials on accumulation of hexakis (2-methoxyisobutylisonitrile) technetium (I) in cultured mouse fibroblast. F Nucl Med 1990;31:164653.

7 Piwnica-Worms D, Holman BL. Noncardiac applications of hexakis (alkylisonitrile) technetium-99m complexes. $f$ Nucl Med 1990;31:1166-7.

Med 1990;31:1166-7.
8 Muller SP, Reniers C, Paas M, et al. Tc-99m MIBI and Muller SP, Reniers C, Paas M, et al. Tc-99m MIBI and
Tl-201 uptake in bronchial carcinoma (abstract). $\mathcal{F}$ Nucl Med 1989;30:845.

9 Kao C-H, Wang S-J, Lin W-Y, et al. Differentiation of single solid lesions in the lungs by means of single-photon emission tomography with technetium-99m methoxyisoisonitrile. Eur F Nucl Med 1993;20:249-54.

10 Kostakoglu L, Elahi N, Kiratli P, et al. Clinical validation of the influence of P-glycoprotein on technetium-99msestamibi uptake in malignant tumors. $7 \mathrm{Nucl} \mathrm{Med}$ 1997;38:1003-8.

11 Nishiyama Y, Kawasaki Y, Yamamoto Y, et al. Technetium99m-MIBI and thallium-201 scintigraphy of primary lung $99 \mathrm{~m}-\mathrm{MIBI}$ and thallium-201 scintigrap

12 Wang H, Maurea S, Mainolfi C, et al. Tc-99m MIBI scintigraphy in patients with lung cancer. Comparison with CT and fluorine-18 FDG PET imaging. Clin Nucl Med 1997;22:243-9. 